\title{
Questionário Sociocognitivo para Deixar de Fumar: Construção de um Instrumento baseado na Teoria do Comportamento Planeado
}

\author{
Socio-Cognitive Questionnaire about Smoking Cessation: Construction of an \\ Instrument based on the Theory of Planned Behavior
}

Fernando Afonso ${ }^{1}$ e M. Graça Pereira ${ }^{1}$

\begin{abstract}
Resumo
O Questionário do Comportamento Planeado para Deixar de Fumar (QCP-DF) é um instrumento que avalia crenças relacionadas com a cessação tabágica. Este artigo apresenta as características psicométricas do questionário. Vinte e cinco fumadores participaram no estudo qualitativo e 224 no estudo de validação. Foram efetuadas entrevistas para elicitar crenças em relação à cessação tabágica que foram posteriormente analisadas com base na análise de conteúdo. Desta forma, foi construído um questionário de acordo com a Teoria do Comportamento Planeado. A validação do questionário revelou uma boa fidelidade para as subescalas e boa validade de constructo, correlacionando-se com as representações associadas ao tabaco bem como validade descriminante com o teste de Fagerström para a dependência nicotínica. Assim, o QCP-DF parece ser um instrumento útil no planeamento de programas de cessação tabágica.
\end{abstract}

Palavras-chave: tabagismo, teoria do comportamento planeado, construção de escala

\begin{abstract}
The Planned Behavior Questionnaire to Quit Smoking (PBQ-QS) is an instrument that measures beliefs related to smoking cessation. This paper presents the psychometric characteristics of QCP-DF. Twenty five smokers participated in the qualitative study and 224 in validation study. Interviews were done in order to elicit beliefs related to smoking cessation that were later analyzed using content analysis. By this process, a questionnaire was constructed according to the Theory of Planned. The validation of the questionnaire revealed good reliability for the subscales and good construct validity showing a correlation with tobacco representations and good discriminant validity with the Fagerström Test for Nicotine Dependence. The QCPDF seems to be a useful instrument to include in smoking cessation programs.
\end{abstract}

Keywords: tobacco, theory of planned behavior, scale construction

${ }^{1}$ Universidade do Minho. Escola de Psicologia, Campus de Gualtar, 4710-057 Braga, Portugal. E-mail: gracep@psi.uminho.pt 


\section{Introdução}

A literatura mostra que fumar é uma das principais causas evitáveis de doença, incapacidade e morte prematura, em países desenvolvidos, a nível mundial (World Health Organization, 2008). De acordo com o último relatório em Portugal (Programa Nacional para a Prevenção e Controlo do Tabagismo, 2013) estima-se que o consumo de tabaco tenha sido responsável por $1 \mathrm{em}$ cada 10 mortes verificadas na população adulta e por cerca de 1 em cada 4 mortes verificadas na população dos 45 aos 59 anos.

Os fumadores deixam de fumar por vários motivos, nomeadamente, questões de saúde (e.g. doenças do foro respiratório), questões psicossociais (e.g. pressão de família e cônjuges, pares, razões económicas) (Rossaneis \& Machado, 2012) e psicológicas (e.g. melhor autoestima) (Duarte, Campos, Monteiro, \& Oliveira, 2015).

No processo de deixar de fumar, os fumadores, podem apresentar dependência física à nicotina acompanhada de sintomas físicos, alterações de humor/ansiedade quando tentam deixar de fumar, especialmente os que fumam mais cigarros por dia (Sattler \& Cade, 2013). Estudos revelam que fumadores com elevada dependência à nicotina apresentam mais resistência ao tratamento para deixar de fumar e necessitam de uma intervenção mais complexa, combinando métodos cognitivo-comportamentais e métodos alternativos (por ex., estratégias de promoção da saúde física e mental) (Cini, Flores, \& Pannuti, 2012; Duarte et al., 2015). Além da dependência física também estão presentes dificuldades de dimensão cognitiva e emocional, tais como a associação de sentimentos positivos ao uso do cigarro (sensação de calma), alívio de sentimentos negativos (ansiedade, agitação motora, tristeza), dependência psicológica (dado o cigarro ser percecionado como "um amigo") e falta de motivação para deixar de fumar (Echer, Almeida, Lukasewicz, \& Lucena, 2011; Souza, Crippa, Pasian, \& Martinez, 2009; Veloso et al., 2011).

A teoria do comportamento planeado (TCP) (Ajzen, 1985, 1991) surge como uma teoria alternativa para justificar como as influências sobre um indivíduo determinam a sua decisão relativa a um determinado comportamento no âmbito da saúde. Esta teoria propõe que a intenção de uma pessoa para realizar um comportamento é o determinante fundamental desse comportamento, porque reflete o nível de motivação da pessoa para implementar esforços em relação à ação (Courneya \& McCauley, 1995). Para a TCP, as intenções para a implementação de um comportamento envolvem três aspetos: 1) as atitudes face ao comportamento; 2) as normas subjetivas e 3) e o controlo comportamental percebido (CCP) (Ajzen, 1991). Segundo a TCP, as atitudes face ao comportamento, as normas subjetivas e o CCP são variáveis que estão associadas a um comportamento e permitem predizer a intenção para a execução desse mesmo comportamento. Assim, a atitude reflete-se numa avaliação positiva ou negativa do comportamento a executar (por ex., deixar de fumar será bom/mau; útil/inútil; cómodo/incomodo; saudável/perigoso); a norma subjetiva espelha a perceção da pressão/influência que os indivíduos podem sentir para executar ou não o comportamento (e.g. a minha família pensa que eu devo/não devo deixar de fumar); e o controlo comportamental percebido refere-se à facilidade, ou dificuldade, em implementar o comportamento (por ex., deixar de fumar nos próximos seis meses será fácil/difícil) (Courneya et al., 1995). Um estudo realizado por Dohnke, Weiss-Gerlach, e Spies (2011), com o objetivo de analisar os aspetos envolvidos na intenção para deixar de fumar, revelou a importância das normas subjetivas na motivação para deixar de fumar tanto em homens como mulheres.

Segundo Ajzen (2002), estes três constructos designam-se de medidas diretas e têm na sua base crenças (medidas indiretas) que o sujeito possui acerca do comportamento. As crenças são um elemento central na TCP e são consideradas bases sólidas a nível cognitivo e afetivo para a construção das atitudes, normas subjetivas e CCP. Estas crenças podem ser: comportamentais (referem-se aos resultados prováveis da realização de determinado comportamento); normativas (referem-se à perceção do que os outros significativos pensam acerca do que o indivíduo deve ou não deve fazer) e de controlo (referem-se 
aos recursos, e obstáculos que o indivíduo perceciona para a realização do comportamento).

No entanto, mesmo considerando medidas diretas e indiretas, a TCP apresenta lacunas, nomeadamente pelo facto de não explicar como é que as intenções dão origem à mudança de comportamento (Sniehotta, 2009). Assim, foram incluídos mais dois processos autorregulatórios no modelo, para explicar como as intenções são implementadas: Planeamento da Ação e Planeamento do Coping. Este planeamento pretende considerar quais as respostas possíveis face a situações futuras ("se acontecer A eu faço B") e pode ser considerado um antecessor do comportamento a atingir (Gollwitzer, 1999). O Planeamento da Ação é o processo pelo qual se define o quando, onde, e como agir, de modo a implementar as intenções comportamentais (Gollwitzer, 1999). Para Gollwitzer (1999), o processo designa-se por implementação de intenções ou planos. Se-Então ("If-Then Plans"). Ao planear, os sujeitos têm mais probabilidade de agir de acordo com as suas intenções, podendo antecipar oportunidades e organizar respostas e implementar o comportamento mais rapidamente (Gollwitzer, 1999). Este processo cognitivo permite definir quais os aspetos, internos ou externos, a ter em conta pelo facto de existir uma antecipação prévia e uma representação mental que liga as respostas às situações (Sniehotta, Scholz, \& Schwarzer, 2006). Ao nível da cessação tabágica, o resultado do planeamento da ação dependerá do nível da motivação e intenção para deixar de fumar (Armitage \& Arden, 2008; Hilberink, Jacobs, Schlösser, Grol, \& de Vries, 2006). É possível que alguns projetos pessoais considerados como difíceis de concretizar como deixar de fumar, possam beneficiar do planeamento de ação, identificando como, quando e onde mudar o comportamento (action planning) e estratégias de coping (coping planning), auxiliando o indivíduo na implementação das suas intenções (de Vries, Eggers, \& Bolman, 2013; Dalum, Schaalma, \& Kok, 2012; Michie, Churchill, \& West, 2011; Sutton, 2010).

A literatura tem mostrado a importância dos constructos da TCP não só ao nível dos comportamentos aditivos; mas também do uso de protetor solar (Rodrigues, 2009), redução do consumo de gorduras (Armitage, 2004); atividade física (Ferreira \& Pereira,
2016) e auto-monitorização da glicose (Costa \& Pereira, 2015) em pacientes com diabetes tipo 2 os resultados evidenciaram a pertinência da inclusão de estratégias cognitivas para promover a mudança comportamental.

A TCP integra-se numa perspetiva sociocognitiva, na medida em que contempla fatores sociais (e.g., normas subjetivas) e cognitivos (e.g., intenção, atitudes, controlo percebido, planeamento). De acordo com a TCP, atitudes mais fortes têm uma maior probabilidade de predizer as intenções (Fila \& Smith, 2006). Pelo contrário, os indivíduos que creem não ter recursos ou oportunidades para realizar um determinado comportamento (controlo comportamental percebido) têm menor probabilidade de formar intenções comportamentais para a sua realização, independentemente de manterem atitudes favoráveis em relação ao comportamento e acreditarem que os outros significativos aprovariam a realização desse comportamento.

Assim, se um fumador quer deixar de fumar e considerar que isso é benéfico para a sua saúde, principalmente ao nível da capacidade física e pulmonar (atitude), se a família apoiar esta decisão como sendo muito adequada disponibilizando-se para ajudar (norma subjetiva) e o fumador apresentar uma baixa perceção da sua competência para deixar de fumar (perceção de baixo controlo do comportamento), o fumador pode apresentar uma baixa intenção para deixar de fumar, apesar de apresentar atitudes e normas subjetivas favoráveis.

Quanto ao poder preditivo envolvido na mudança de comportamento, a TCP tem sido aplicada a diversos comportamentos (Côté, Godin, \& Gagué, 2004; Hill, Boudreau, Amyot, Déry, \& Godin, 1997; Moan \& Rise, 2005). Num estudo efetuado por Armitage e Conner (2001), em que participaram fumadores em fase de cessação tabágica, verificou-se que os fumadores, em primeiro lugar, pensavam no que beneficiariam se deixassem de fumar (atitudes face ao comportamento), bem como o que teriam que fazer para o conseguir (CCP), estando conscientes de quem aprovaria ou não a sua decisão (normas subjetivas). Estes resultados em relação ao poder preditivo do modelo, também vão ao encontro de outros estudos (French et al., 2005; Kraft, Rise, No43 Vol.1 1 107-122 2017 
Sutton, \& Røysamb, 2005) que mostraram a importância das atitudes afetivas face às instrumentais no que respeita à intenção para fazer atividade física. Quando se trata de aumentar a motivação para deixar de fumar, também pode ser importante para o indivíduo concentrar-se mais nas crenças afetivas (sentimentos positivos e negativos), em vez de se concentrar apenas nas crenças instrumentais (e.g. vantagens/desvantagens em deixar de fumar).

Deixar de fumar pode ser considerado um comportamento planeado dado que o processo está relacionado com uma decisão racional e com a implementação de processos autorregulatórios (Kovac, Rise, \& Moan, 2010). É frequente os indivíduos falharem as suas intenções porque a motivação parece não ser suficiente para assegurar a mudança de comportamento (Sheeran, Milne, Webb, \& Gollwitzer, 2005). Na verdade, ao nível dos comportamentos aditivos, muitas tentativas resultam em fracasso antes do sucesso (Piasecki, Fiore, McCarthy, \& Baker, 2002). Norman, Conner, e Bell (2000) propuseram a inclusão de um conjunto de processos autorregulatórios, como a elaboração de planos detalhados, com vista a aumentar a probabilidade de sucesso na desabituação tabágica. Por sua vez, Armitage e Arden (2008) verificaram a importância da elaboração de planos de ação que terão mais impacto, e estarão mais relacionados, com a predição de sucesso se forem implementados em estádios precoces do comportamento tabágico. Outros estudos revelaram a importância de tentativas prévias para deixar de fumar na predição do sucesso em deixar de fumar (Moan \& Rise, 2005; Rise, Kovac, Kraft, \& Moan, 2008).

Considerando que i) fumar está associado a elevados níveis de morbilidade e mortalidade a nível mundial; ii) a importância de perceber melhor os processos psicológicos envolvidos na cessação tabágica; iii) a utilidade da TCP para explicar a motivação e a intenção, bem como os processos autorregulatórios envolvidos na cessação tabágica; iv) e dado não existir nenhum instrumento para a população portuguesa, optouse por construir um instrumento que permita avaliar estes processos, para no futuro se desenharem intervenções adequadas às necessidades dos fumadores que desejam deixar de fumar. O objetivo deste estudo focou-se assim Revista Iberoamericana de Diagnóstico y Evaluación - e Avaliação Psicológica. RIDEP · No43 · Vol.1 · 107-122 · 2017 na construção de um instrumento que avaliasse as crenças acerca do comportamento tabágico, tendo por base o modelo teórico da Teoria do Comportamento Planeado.

\section{Método}

\section{Participantes}

No estudo qualitativo, na criação do questionário, participaram 25 fumadores $(60 \%$ sexo feminino e $40 \%$ do sexo masculino), e numa segunda fase que incluiu a validação do instrumento, participaram 224 fumadores (52.7\% sexo feminino e $47.3 \%$ do sexo masculino). A maioria dos fumadores fumava há mais de 3 anos (76.8\%) e a idade mais frequente para começar a fumar situou-se nos 16 anos (18.3\%). Do total da amostra, $60.3 \%$ dos participantes efetuou, pelo menos uma tentativa para deixar de fumar, $96 \%$ nunca frequentou qualquer tratamento para deixar de fumar e $39.7 \%$ tinham um parceiro que fumava. Em relação ao estado civil, $37.9 \%$ dos participantes eram casados, $61.6 \%$ tinham como habilitações literárias o ensino secundário e $54.5 \%$ provinham de meio urbano. $\mathrm{O}$ grupo mais numeroso, $41.1 \%$, pertencia ao intervalo de idades entre 21-30 anos logo seguido do intervalo 31-40 anos com $33.5 \%$. Os critérios de inclusão para a amostra foram: ser maior de 18 anos e ser fumador de pelo menos 10 cigarros/dia.

\section{Instrumentos}

- Questionário Sociodemográfico e Clínico (Pereira \& Afonso, 2010): tinha como objetivo recolher informações para caracterizar os participantes da amostra ao nível de: estado civil, sexo, proveniência, habilitações literárias, tipo e quantidade de cigarros consumidos, tentativas para deixar de fumar, tempo sem fumar, frequência de algum tipo de programa para deixar de fumar, e o parceiro fumar/não fumar.

- Brief Illness Perception Questionnaire (Brief-IPQ), (Broadbent, Petrie, Main, \& Weinman, 2006; versão portuguesa de Figueiras \& Alves, 2007). Este instrumento é constituído por nove itens que permitem avaliar as representações emocionais e cognitivas da doença. O Brief-IPQ utiliza uma escala likert (0 a 10) com exceção da questão 9 (questiona o paciente 
acerca das três causas mais importantes para a sua doença, mas que não foi incluída neste estudo). Os itens descrevem as seguintes representações: Consequências (item 1), Duração (item 2), Controlo Pessoal (item 3), Controlo do Tratamento (item 4) Identidade (item 5), Preocupação (item 6), Compreensão (item 7), Resposta Emocional (item 8) e Causas da doença (item 9). Cinco dos itens avaliam as representações cognitivas de doença: Consequências, Duração, Controlo Pessoal, Controlo do Tratamento e Identidade. Dois dos itens avaliam as representações emocionais: Preocupação e Emoções. Um item avalia a Compreensão da doença. Dado que as subsubescalas do instrumento são constituídas por um item não permite efetuar estudos de fidelidade ou validade. De referir que no questionário se substituiu o termo "doença" por "consumo tabágico". Os itens 3, 4 e 7 são invertidos. Um valor elevado em cada item representa maior intensidade no tipo de crença avaliada.

- Fagerström Test for Nicotine Dependence (Heatherton, Kozlowski, Frecker, \& Fagerstrom, 1991; versão portuguesa de Ferreira, Quintal, Lopes, \& Taveira, 2009). Para o presente estudo foi utilizada a versão de 6 itens com vista a avaliar o grau presente de dependência de nicotina nos fumadores. O valor do $\alpha$ de Cronbach, na presente amostra, foi de .67. Estudos anteriores referem um alfa de Cronbach, de .64 (Heatherton et al., 1991); .51 (Fagerström, 1978) e .55 (Lichtenstein \& Mermelstein, 1986). Neste sentido, decidimos usar o instrumento, dado o valor encontrado na nossa amostra (.67) ser superior ao da versão original e se encontrar muito próximo de .70. Um resultado elevado indica maior dependência de nicotina (0-4 pontos: dependência baixa; 4-7 pontos: dependência moderada; > 7 pontos: dependência elevada).

\section{Procedimento}

Relativamente aos constructos da TCP, o questionário sociocognitivo foi construído com base nas diretrizes propostas por Ajzen (2002) e Francis et al. (2004). Assim, numa primeira fase do estudo, foi elaborada uma entrevista para a elicitação de crenças aplicada a uma amostra de conveniência constituída por 25 fumadores. O guião da entrevista era composto por 7 questões que contemplavam a elicitação das crenças de comportamento (1.Quais são as vantagens que vê em deixar de fumar?, 2. Quais são as desvantagens que vê em deixar de fumar?, 3. Quem aprovaria que deixasse de fumar?, 4. Quem não aprovaria o seu comportamento de deixar de fumar, 5. O que poderá facilitar deixar de fumar?, 6. $O$ que poderá tornar mais difícil deixar de fumar?, 7. Para responder à questão anterior pensou nas barreiras que podem tornar difícil deixar de fumar?).

Os fumadores que participaram da fase de elicitação de crenças tinham entre 22 e 60 anos. Os participantes foram selecionados num Hospital Central de Braga. A participação foi voluntária. Após o consentimento informado, os participantes foram entrevistados de forma individual e as entrevistas transcritas para papel. As entrevistas decorreram ao longo de uma semana e cada uma tinha a duração aproximadamente de 30 minutos.

\section{Análise de Dados}

As entrevistas foram analisadas através da análise de conteúdo (Bardin, 2008) que identificou uma lista de crenças significativas (Quadro 1) que permitiram a elaboração dos itens do Questionário Sociocognitivo.

Para o estudo da fidelidade do instrumento procedeu-se à análise da consistência interna realizada com recurso ao alfa de Cronbach. Para o estudo da validade, foi realizada uma análise fatorial através do método dos componentes principais com rotação varimax. Para avaliar a validade de constructo foi avaliada a relação entre as subescalas do Questionário do Comportamento Planeado para Deixar de Fumar (QCP-DF) e as subescalas das representações face ao tabaco (Brief-IPQ) e, para a validade discriminante, foi avaliada a relação com o teste de dependência nicotínica (FT).

\section{Resultados}

Os resultados deste estudo podem ser agrupados em três momentos: 1) análise de conteúdo para análise das respostas dos participantes (fase de elicitação de crenças); 2) transformação das respostas em itens, e 3) 
construção e validação do instrumento tendo em conta as medidas diretas das atitudes, normas subjetivas, controlo percebido, planeamento de ação e de coping.

\section{Análise de Conteúdo}

As respostas foram analisadas para permitir a construção de categorias relacionadas com as crenças normativas e de controlo (Ajzen, 2002) de modo a permitir a construção dos itens referentes às crenças do questionário (Quadro 1). Ao nível das crenças de comportamento perguntou-se aos participantes acerca das vantagens e desvantagens de deixar de fumar (1.Quais são as vantagens que vê em deixar de fumar?; 2. Quais são as desvantagens que vê em deixar de fumar?). Verificou-se (Quadro 1) que os participantes referiram, como vantagens, uma melhoria da saúde (80\%), uma melhoria na condição económica (64\%), uma melhoria no seu hálito (40\%) e uma convivência com pessoas que não fumam sem risco de incómodo (48\%). Em relação às desvantagens, os participantes referiram 0 aumento do stress, nervosismo ou ansiedade $(56 \%)$, ver amigos a fumar (32\%) ou tomar café (40\%).

Em relação à construção das crenças normativas elaboraram-se duas questões que avaliavam quem aprovava e desaprovava deixar de fumar (3. Quem aprovaria que deixasse de fumar?; 4. Quem não aprovaria o seu comportamento de deixar de fumar?). Os participantes indicaram, basicamente, duas figuras representativas: a família (96\%) onde se inclui os parceiros/companheiros; e o médico (64\%). Em relação às pessoas que desaprovariam deixar de fumar, os participantes indicaram ninguém (20\%) e eventualmente alguns amigos (28\%).

As crenças de controlo foram elaboradas a partir de duas questões que avaliavam as facilidades, e dificuldades/obstáculos, em deixar de fumar (5. O que poderá facilitar deixar de fumar? 6. O que poderá tornar mais difícil você deixar de fumar?). Como facilitadores de deixar de fumar foi referida a motivação para deixar de fumar (60\%), ter um filho (ou engravidar) (40\%), as pessoas chegadas também deixarem de fumar $(36 \%)$ e ter problemas relacionados com a saúde (68\%). Como dificuldades/obstáculos foram referidos, o stress, ansiedade e nervosismo (56\%), Revista Iberoamericana de Diagnóstico y Evaluación - e Avaliação Psicológica. RIDEP · No43 · Vol.1 · 107-122 · 2017 ver amigos a fumar (34\%), e tomar café (40\%). No que respeita a ultrapassar possíveis barreiras (7. Para responder à questão anterior pensou nas barreiras que podem tornar difícil deixar de fumar?) foi referido o que fazer para organizar o tempo para não fumar (44\%), o que fazer para não fumar (52\%), o que fazer para não comprar cigarros $(34 \%)$, o que fazer se algo interferir nos planos para não fumar (28\%) e o que fazer para lidar com possíveis obstáculos e/ou dificuldades $(28 \%)$.

\section{Transformação da Elicitação de Crenças em Itens}

Nesta fase, as crenças dos participantes foram transformadas em itens: crenças de comportamento, crenças normativas e crenças de controlo segundo as diretrizes de Ajzen (2002) tendo em conta as respostas com mais relevância (e.g. Crenças de comportamento: Deixar de fumar nos próximos 6 meses permite-me melhorar a minha saúde; Crenças Normativas: A minha família pensa que eu devia/não devia deixar de fumar nos próximos 6 meses; Crenças de Controlo: O stress torna muito difícil/ fácil deixar de fumar nos próximos 6 meses). Ao nível das medidas diretas, foram tidas em consideração a intenção para deixar de fumar (inclui um item que avaliou a intenção em relação ao comportamento para deixar de fumar), as atitudes em relação ao comportamento para deixar de fumar (4 itens avaliada pelos participantes em uma escala de cinco pontos, como mau-bom, inútil-útil, incómodo-cómodo, perigoso-saudável), normas subjetivas ( 5 itens para avaliar a opinião de outros significativos), controlo percebido (4 itens foram incluídos que avaliam a capacidade percebida dos participantes para deixar de fumar), planeamento de ação (4 itens para avaliar se os fumadores tinham um plano para deixar de fumar e planeamento de coping (5 itens para avaliar como lidar com as dificuldades se deixassem de fumar).

\section{Estudos de Validade}

Procedeu-se a um estudo da análise fatorial com rotação Varimax com noves fatores, seguindo os nove constructos da TCP (Ajzen, 2002) cujos resultados tendo por base a saturação dos itens acima de .30 (Iacobucci, 2001) não 
Quadro 1. Elicitação de Crenças

\begin{tabular}{|c|c|c|}
\hline Crenças de comportamento & $\mathbf{n}$ & $\%$ \\
\hline \multicolumn{3}{|l|}{ Vantagens } \\
\hline Uma melhoria da saúde & 20 & 80 \\
\hline Uma melhoria na condição económica & 16 & 64 \\
\hline Uma melhoria no meu hálito & 10 & 40 \\
\hline $\begin{array}{l}\text { Uma convivência com pessoas, que não fumam, sem risco de } \\
\text { incómodo }\end{array}$ & 12 & 48 \\
\hline \multicolumn{3}{|l|}{ Desvantagens } \\
\hline Aumento do stresse, nervosismo ou ansiedade & 14 & 56 \\
\hline Ver amigos a fumar & 8 & 32 \\
\hline Tomar café & 10 & 40 \\
\hline \multicolumn{3}{|l|}{ Crenças normativas } \\
\hline \multicolumn{3}{|l|}{ Pessoas que aprovariam deixar de fumar: } \\
\hline Família (parceiros/companheiros) & 24 & 96 \\
\hline Médico & 16 & 64 \\
\hline \multicolumn{3}{|l|}{ Pessoas que desaprovariam deixar de fumar: } \\
\hline Ninguém & 5 & 20 \\
\hline Alguns amigos & 7 & 28 \\
\hline \multicolumn{3}{|l|}{ Crenças de controlo } \\
\hline \multicolumn{3}{|l|}{ Facilitadores para deixar e fumar } \\
\hline Motivação para deixar de fumar & 15 & 60 \\
\hline Ter um filho (ou engravidar) & 10 & 40 \\
\hline As pessoas chegadas também deixarem de fumar & 9 & 36 \\
\hline Ter problemas relacionados com a saúde & 17 & 68 \\
\hline \multicolumn{3}{|l|}{ Barreiras para deixar de fumar } \\
\hline Stresse, ansiedade e nervosismo & 14 & 56 \\
\hline Ver amigos a fumar & 8 & 34 \\
\hline Tomar café & 10 & 40 \\
\hline \multicolumn{3}{|l|}{ Ultrapassar barreiras } \\
\hline $\begin{array}{l}\text { O que fazer para organizar o tempo para não fumar } \\
\text { O que fazer para não fumar }\end{array}$ & 11 & 44 \\
\hline O que fazer para não comprar cigarros & 13 & 52 \\
\hline O que fazer se algo interferir nos planos para não fumar & 8 & 34 \\
\hline $\begin{array}{l}\text { O que fazer para lidar com possíveis obstáculos e/ou } \\
\text { dificuldades }\end{array}$ & 7 & 28 \\
\hline
\end{tabular}

foram satisfatórios. A versão final contempla sete fatores cujos itens saturam acima de .40, e fazem sentido teórico segundo a TCP com alfas de Cronbach superiores a .70, explicando no seu conjunto, $65.82 \%$ da variância total dos resultados. A versão final do questionário mostra uma organização dos itens em sete fatores. Assim, Fator 1: Planeamento do Coping e Ação (9 itens: 11a, 11b, 11c, 11d, 12a, 12b, 12c, 12d, 12e); Fator 2: Crenças de Comportamento (8 itens: $4 \mathrm{a}, 4 \mathrm{~b}, 4 \mathrm{c}$, 4d, 5a, 5b, 5c, 5d); Fator 3: Normas Subjetivas (4 itens: 6a, 6c, 6d e 6e tendo sido eliminado o item 6b); Fator 4: Controlo Comportamental Percebido (4 itens: 8a, 8b, 8c, 8d); Fator 5: Atitudes face ao Comportamento (4 itens: 3a, 3b, 3c, 3d); Fator 6: Crenças Normativas e Crenças de Controlo (7 itens: 7c, 9b, 9c, 9d, 10a, 10b, 10c) e Fator 7: Intenção (um item: item 2) (Quadro 2).

Ao nível das medidas indiretas, resultados mais elevados correspondem a crenças normativas e de controlo bem como crenças comportamentais mais fortes e ao nível das medidas diretas, a maior 
Quadro 2. Estrutura Fatorial do Questionário do Comportamento Planeado para Deixar de Fumar (medidas indiretas e diretas) $(\mathrm{N}=224)$

\begin{tabular}{|c|c|c|c|c|c|c|c|}
\hline \multirow[b]{2}{*}{ Itens } & \multicolumn{7}{|c|}{ Fatores } \\
\hline & $\begin{array}{l}\text { Planeamento } \\
\text { Coping/Ação }\end{array}$ & $\begin{array}{c}\text { Crenças } \\
\text { Comportamento }\end{array}$ & $\begin{array}{c}\text { Normas } \\
\text { subjetivas }\end{array}$ & $\begin{array}{l}\text { C.Comportamental } \\
\text { Percebido }\end{array}$ & $\begin{array}{c}\text { Atitudes } \\
\text { Comortamentais }\end{array}$ & $\begin{array}{c}\text { Crenças } \\
\text { normativas/ } \\
\text { controlo }\end{array}$ & Intenção \\
\hline 2. & & & & & & & .40 \\
\hline $3 a$. & & & & & .89 & & \\
\hline $3 \mathrm{~b}$. & & & & & .81 & & \\
\hline 3c. & & & & & .80 & & \\
\hline $3 \mathrm{~d}$. & & & & & .83 & & \\
\hline 4a) & & .53 & & & & & \\
\hline $4 \mathrm{~b})$ & & .51 & & & & & \\
\hline $4 c)$ & & .45 & & & & & \\
\hline 4d) & & .41 & & & & & \\
\hline 5a) & & .80 & & & & & \\
\hline $5 \mathrm{~b})$ & & .70 & & & & & \\
\hline $5 c)$ & & .82 & & & & & \\
\hline 5d) & & .77 & & & & & \\
\hline 6a) & & & -.65 & & & & \\
\hline $6 c)$ & & & -.55 & & & & \\
\hline 6d) & & & -.67 & & & & \\
\hline 6e) & & & -.73 & & & & \\
\hline 7c) & & & & & & .50 & \\
\hline 8a) & & & & .64 & & & \\
\hline $8 \mathrm{~b})$ & & & & .82 & & & \\
\hline 8c) & & & & .87 & & & \\
\hline 8d) & & & & .49 & & & \\
\hline $9 \mathrm{~b})$ & & & & & & .69 & \\
\hline 9c) & & & & & & .51 & \\
\hline 9d) & & & & & & .62 & \\
\hline 10a) & & & & & & -.63 & \\
\hline $10 b)$ & & & & & & -.50 & \\
\hline $10 \mathrm{c})$ & & & & & & -.73 & \\
\hline 11a) & .80 & & & & & & \\
\hline $11 \mathrm{~b})$ & .47 & & & & & & \\
\hline 11c) & .60 & & & & & & \\
\hline 11d) & .69 & & & & & & \\
\hline 12a) & .86 & & & & & & \\
\hline $12 \mathrm{~b})$ & .87 & & & & & & \\
\hline $12 \mathrm{c})$ & .87 & & & & & & \\
\hline $12 \mathrm{~d})$ & .80 & & & & & & \\
\hline 12e) & .76 & & & & & & \\
\hline
\end{tabular}

intenção, atitudes mais positivas, maior pressão dos outros significativos (normas subjetivas), maior perceção de controlo percebido e maior planeamento da ação/coping, para deixar de fumar.

\section{Fidelidade}

Para análise de consistência interna dos itens procedeu-se ao cálculo do alfa de Cronbach. Para a subescala Planeamento do Coping e da Ação obteve-se um alfa de .91, a subescala Normas Subjetivas obteve um alfa de .68, a subescala Controlo Comportamental Percebido obteve um alfa de .81, a subescala Atitudes face ao Comportamento obteve um alfa de .91 e a subescala Intenção não apresentou valor de alfa por ser constituída por um item. Os alfas das subescalas das medidas indiretas foram: Crenças de Comportamento: .87, e a subescala Crenças de Controlo/Normativas obteve um alfa de .75.

\section{Validade de Construto}

Para a validade de constructo foi avaliada a relação entre as subescalas do QCP-DF (medidas diretas) e o Brief-IPQ tendo em consideração que os itens controlo pessoal, controlo do tratamento e compreensão são cotados de forma invertida (Quadro 3). Verificaram-se correlações significativas entre as variáveis sociocognitivas e as representações face ao tabaco. Assim, verificou-se uma correlação positiva entre a intenção de deixar de fumar (QCP-DF) e as 
consequências, controlo pessoal, identidade, preocupação e resposta emocional face ao tabaco (Brief-IPQ), ou seja, maior intenção para deixar de fumar encontrou-se associada à perceção de mais consequências do tabaco, menor controlo sobre o ato de fumar, mais sintomas associados ao tabaco, e maior impacto emocional do tabaco. Verificou-se também uma correlação negativa com a duração e o controlo do tratamento, ou seja, maior intenção de deixar de fumar está associada a menor duração do hábito de fumar e perceção de maior controlo sobre a eficácia do tratamento.

Ao nível das atitudes para deixar de fumar verificaram-se correlações positivas com as consequências, duração e identidade, ou seja, atitudes mais positivas para deixar de fumar estão associadas à perceção de mais consequências, duração prolongada do consumo tabágico, e mais sintomas associados ao hábito tabágico. Além disso, verificaram-se correlações negativas com o controlo pessoal, sendo que atitudes mais positivas do fumador estão associadas à perceção de maior controlo pessoal para deixar de fumar.

No que toca às normas subjetivas foram encontradas correlações positivas com a duração e o controlo de tratamento, e correlações negativas com as consequências, controlo pessoal, preocupação e compreensão. Assim, a perceção da pressão para deixar de fumar (outros significativos) é maior, quanto maior for a duração do hábito tabágico, maior o controlo pessoal sobre o comportamento de fumar, menores as consequências percecionadas, menor a perceção de eficácia do tratamento, menor a preocupação e maior a compreensão sobre o comportamento de fumar.

No que respeita ao controlo comportamental percebido, este é maior quanto menor for a perceção de controlo sobre o tratamento para deixar de fumar, das consequências, dos sintomas e do impacto emocional de fumar; e maior a perceção de controlo pessoal.

Por fỉm, em relação ao planeamento do coping/ação, encontraram-se correlações positivas com a preocupação e correlações negativas com a duração e controlo de tratamento.

Assim, uma perceção mais forte sobre o que fazer e como fazer para deixar de fumar está associada a maior preocupação, maior controlo sobre o tratamento e menor duração do hábito tabágico.

Em suma, de acordo com os resultados, todas as dimensões cognitivas do Brief-IPQ estão relacionados com os constructos da TCP mostrando a relação entre as representações do comportamento de fumar e as crenças acerca do comportamento planeado, para deixar de fumar.

Quadro 3. Resultados da Correlação de Pearson entre as Representações do Tabaco (B-IPQ) as variáveis sociocognitivas (medidas diretas) da Teoria do Comportamento Planeado $(N=224)$

\begin{tabular}{|c|c|c|c|c|c|}
\hline $\begin{array}{l}\text { Representações } \\
\text { (B-IPQ) }\end{array}$ & Intenção & $\begin{array}{l}\text { Atitudes } \\
\text { face } \\
\text { Comporta } \\
\text { mento } \\
\end{array}$ & $\begin{array}{l}\text { Normas } \\
\text { Subjetivas }\end{array}$ & $\begin{array}{c}\text { C. C. } \\
\text { Percebido }\end{array}$ & $\begin{array}{l}\text { Planeamento } \\
\text { Coping/Ação }\end{array}$ \\
\hline Consequências & $.27^{* *}$ & $.13^{*}$ & $-.14^{*}$ & $-.22^{* *}$ & .09 \\
\hline Duração & $-.26^{* *}$ & $.25^{* *}$ & $.17^{* *}$ & -.12 & $-.34^{* *}$ \\
\hline Controlo Pessoal & $.31^{* *}$ & $-.16^{*}$ & $-.38^{* *}$ & $-.46^{* *}$ & .04 \\
\hline Controlo Tratam. & $-.23^{* *}$ & .03 & $.21^{* *}$ & $.26^{* *}$ & $-.20^{* *}$ \\
\hline Identidade & $.25^{* *}$ & $.17^{* *}$ & -.01 & $-.26^{* *}$ & .02 \\
\hline Preocupação & $.40^{* *}$ & .02 & $-.24^{* *}$ & -.06 & $.42^{* *}$ \\
\hline Compreensão & -.09 & .12 & $-.17^{* *}$ & -.01 & -.05 \\
\hline $\begin{array}{l}\text { Resposta } \\
\text { Emocional }\end{array}$ & $.14^{*}$ & -.05 & .00 & $-.17^{*}$ & .08 \\
\hline
\end{tabular}




\section{Validade Discriminante}

Tendo por base os resultados da dependência nicotínica avaliados a partir do Teste de Fagerstrom, verificou-se uma correlação negativa com o controlo comportamental percebido ( $r=$ $.300, p \leq .001)$ e nenhuma relação com as restantes subescalas do questionário, como seria de esperar. Assim, apenas os fumadores que percecionaram ter menos controlo revelaram ter dependência nicotínica. Nas restantes subescalas, os fumadores com resultado elevado no QCP-DF, não apresentaram dependência nicotínica.

\section{Discussão}

O objetivo principal deste estudo foi a construção de um instrumento sociocognitivo com base na TCP. Assim, iniciamos este processo com um estudo qualitativo que permitiu avaliar as crenças de comportamento, normativas e de controlo em relação ao tabaco que foram posteriormente testadas, num estudo quantitativo. Foram contempladas as medidas diretas neste instrumento, no sentido de melhor compreender os fatores psicológicos que fundamentam a intenção para mudar um comportamento voluntariamente.

No que diz respeito às características psicométricas, o instrumento organizou-se em sete fatores: Intenção (1 item), Atitudes face ao comportamento (4 itens), com alfa de .91; Crenças de Comportamento ( 8 itens) com alfa de .87; Controlo Comportamental Percebido (4 itens) com alfa de .81; Normas Subjetivas (4 itens) com alfa de .68; Crenças Normativas/Controlo (7 itens) com alfa de .75; Planeamento do Coping e Ação (9 itens) com alfa de .91; apresentando, portanto, bons valores de consistência interna. A subescala de normas subjetivas apresenta um alfa mais baixo, mas aceitável dado apresentar apenas 4 itens (Nunally, 1978). Considerando apenas as medidas diretas, a solução fatorial apresentada é semelhante à encontrada por Ferreira e Pereira (2014), na validação do Questionário de Comportamento Planeado, destinado à realização de atividade física, e por Costa e Pereira (2015) na validação do Questionário de Comportamento Planeado, destinado à auto-monitorização da glicose, ambos em pacientes com diabetes tipo 2 .
As soluções fatoriais consistem ambas em cinco fatores: intenção, atitudes, normas subjetivas, controlo comportamental percebido e planeamento ação/coping (Costa \& Pereira, 2015; Ferreira \& Pereira, 2014).

As associações entre o QCP-DF e as representações relativas ao tabaco revelaram que maior intenção para deixar de fumar encontrou-se associada à perceção de mais consequências do tabaco, sintomas associados ao tabaco e maior impacto emocional do tabaco. De facto, a literatura tem evidenciado a preocupação dos fumadores em querer deixar de fumar tendo em consideração a preocupação com o seu estado de saúde (Schane, Ling, \& Glantz, 2010). Também no estudo qualitativo prévio à construção do questionário, foi possível verificar que os participantes consideraram que deixar de fumar melhorava alguns aspetos da sua vida, nomeadamente, a sua saúde (e.g. melhor hálito), a sua condição económica e o convívio com pessoas que não fumam (e que se incomodam com o fumo). $\mathrm{Na}$ verdade, estes resultados vão de encontro às premissas da TCP, já que as consequências do tabaco são determinantes das atitudes que, por sua vez, são determinantes da intenção (Ajzen, 2002), em fumadores (Topa \& Moriano, 2010). Também os sintomas e o impacto emocional podem contribuir para a formação das atitudes relativamente à cessação tabágica e, consequentemente, da intenção de deixar de fumar. Na verdade, no processo de elicitação de crenças, $56 \%$ dos participantes referiram o aumento do stress, nervosismo ou ansiedade como desvantagens associadas ao deixar de fumar, comprovando o impacto emocional do consumo. Constatou-se ainda que maior intenção para deixar de fumar estava associada a menor duração do hábito de fumar, à perceção de menor controlo sobre $\mathrm{o}$ ato de fumar e ao maior controlo sobre a eficácia do tratamento, o que parece intuitivo, na medida em que os indivíduos que fumam podem não percepcionar ter controlo para deixar de fumar por eles próprios, acreditando que o tratamento será eficaz, quanto menor for a duração do hábito tabágico, pelo facto da dependência nicotínica ser menor (Courneya \& McCauley, 1995).

As crenças comportamentais constituem a dimensão antecedente das atitudes na TCP, tendo sido elicitadas através das vantagens e 
desvantagens de deixar de fumar. Os resultados quantitativos mostraram que crenças comportamentais mais fortes para deixar de fumar estavam associadas à perceção de menor controlo pessoal sobre o ato de fumar, perceção de que o tratamento para deixar de fumar será eficaz e menor duração do hábito de fumar. No que diz respeito às atitudes para deixar de fumar, as mais positivas estão associadas à perceção de mais consequências, de maior controlo pessoal para deixar de fumar, duração prolongada do consumo tabágico e mais sintomas associados ao hábito tabágico. De facto, estes resultados são congruentes com o que seria esperado, na medida em que, de acordo com a TCP, as atitudes face a deixar de fumar serão determinadas pelas crenças dos fumadores sobre as consequências de fumar (Ajzen, 1991, 2002). O facto dos fumadores reportarem sintomas devido ao consumo tabágico, provavelmente devido à duração prolongada do consumo, pode não obstante contribuir para a formação de atitudes mais positivas sobre a cessação tabágica. Além disso, o facto desta perceção sobre a cessação tabágica ser positiva aumenta a confiança do fumador acerca do controlo para deixar de fumar. Contudo, as associações entre as crenças comportamentais e as representações sobre o tabaco são opostas às associações entre as atitudes e as respetivas representações, o que poderá dever-se ao facto das atitudes serem fatores determinantes mais próximos da mudança de comportamento, sendo que, quando mais fortes/positivas, maior a probabilidade de predizerem a cessação tabágica (Fila \& Smith, 2006).

As crenças normativas/ de controlo, por sua vez, antecedem as dimensões das normas subjetivas e controlo percebido, tendo sido elicitadas através de questões como quem aprovava e desaprovava deixar de fumar e as facilidades e dificuldades/obstáculos em deixar de fumar, respetivamente. Os resultados mostraram que as crenças mais fortes estão associadas a maior duração do hábito tabágico, à perceção de menor controlo pessoal, maior preocupação e maior impacto emocional do tabaco, o que é intuitivo, na medida em que o fumador acredita que possui menos controlo sobre o ato de fumar, o que gera mais preocupação e, consequentemente, pode afetar emocionalmente. $\mathrm{Na}$ verdade, tem Revista Iberoamericana de Diagnóstico y Evaluación - e Avaliação Psicológica. RIDEP · No43 · Vol.1 · 107-122 2017 sido sugerido que, no sentido de aumentar a motivação para deixar de fumar, torna-se pertinente um maior enfoque nas crenças afetivas do que nas instrumentais (French et al., 2005; Kraft et al., 2005). Conclui-se que, para estes fumadores, a perceção dos recursos internos que possui, bem como o que outros pensam acerca de deixar de fumar, são aspetos importantes na mudança (Ajzen, 2002).

Ao nível das normas subjetivas, a perceção da pressão para deixar de fumar (outros significativos) é maior, quanto maior for a duração do hábito tabágico, maior o controlo pessoal e a compreensão sobre o comportamento de fumar; e, por outro lado, menores as consequências percecionadas, o controlo sobre o tratamento e a preocupação. Uma vez que as normas subjetivas focam-se na pressão dos outros significativos para que o fumador deixe de fumar e, dado que nesta amostra, os fumadores não estão presentemente a tentar deixar de fumar, parece lógico que estes percepcionem maior controlo sobre o ato de fumar, que o tratamento não será eficaz, que compreendem bem o hábito tabágico, que o consumo tem consequências menos negativas, e, por isso, também mostram menos preocupação com esta questão. De facto, os estudos revelam a importância do apoio do parceiro e da família na desabituação tabágica como fonte importante de motivação e suporte (Homish \& Kenneth, 2005; Mermelstein, Cohen, Lichtenstein, Baer, \& Karmack, 1986; Park, Tudiver, Schultz, \& Campbell, 2004). Já o papel do médico aparece como importante na monitorização, promoção da adesão e apoio na desabituação tabágica em contexto clínico (Lancaster, 2011).

No que respeita ao controlo comportamental percebido (CCP), este é maior quanto menor for a perceção de eficácia do tratamento para deixar de fumar, das consequências, dos sintomas e do impacto emocional de fumar; e maior a perceção de controlo pessoal. Sendo o controlo percebido uma dimensão mais intrapessoal do que as normas subjetivas, em que a responsabilidade já é colocada no próprio fumador, é esperado que haja uma maior percepção de controlo do indivíduo sobre $\mathrm{o}$ ato de fumar. $\mathrm{Na}$ verdade, tem sido sugerido que esta dimensão da TCP é semelhante à autoeficácia de Bandura (2004). Neste sentido, a 
perceção quanto à eficácia do tratamento é menor, talvez porque o fumador perceciona que deixar de fumar é uma tarefa que depende apenas de si e que pode fazer quando quiser, sendo que para isso representações como a eficácia do tratamento, as consequências, os sintomas e o impacto emocional não são tão ameaçadoras. De facto, Topa e Moriano (2010) verificaram que o CCP contribuía significativamente para o consumo tabágico, o que vai de encontro à TCP que defende que o controlo comportamental percebido permite predizer diretamente a mudança de comportamento (Ajzen, 1991, 2002).

Finalmente, o planeamento do coping/ação aumentou com a preocupação associada ao tabaco e o controlo sobre o tratamento, e diminuiu com a duração do consumo. Assim, quanto mais preocupado o fumador está com o seu hábito tabágico e maior a perceção de que o tratamento para deixar de fumar será eficaz, mais depressa iniciará um plano para deixar de fumar. Estes resultados vão ao encontro da literatura que mostra a importância da preparação de planos específicos e detalhados para deixar de fumar (de Vries, Eggers, \& Bolman, 2013). Esta preparação por si só mostra-se como preditora da cessação tabágica. Estudos são convergentes com estes resultados mostrando altos índices de planeamento do coping/ação em fumadores com intenção para deixar de fumar no mês seguinte (de Vries et al., 2013). Aliás, a investigação mostra que o planeamento do coping e da ação colmata a lacuna entre a intenção e o comportamento, tornando explícitos aspetos internos e externos que podem interferir com a mudança efetiva de comportamento (de Vries et al., 2013; Sniehotta et al., 2006).

Os resultados revelaram ainda que a dependência nicotínica se relacionava negativamente com o controlo comportamental percebido, ou seja, quanto maior o nível de dependência tabágica menos o fumador considera ser fácil deixar de fumar nos próximos seis meses o que está de acordo com a literatura (Courneya \& McCauley, 1995).

A versão adaptada do QCP-DF comportou-se como um bom instrumento de avaliação das crenças relacionadas no processo de mudança do comportamento tabágico dado apresentar valores de consistência interna satisfatórios, e validade Revista Iberoamericana de Diagnóstico y Evaluación - e Avaliação Psicológica. RIDEP · No43 · Vol.1 · 107-122 · 2017 convergente, correlacionando-se com um questionário de avaliação de representações do tabaco.

Os resultados sugerem a importância das dimensões cognitivas na mudança do comportamento (de Vries et al., 2013) bem como a importância da dimensão motivacional na predição da cessação tabágica (Zhou et al., 2009). Constatou-se, também, que a dependência nicotínica se correlacionava negativamente com o controlo comportamental percebido. A perceção de controlo do comportamento é determinada pelas crenças de controlo e pelo poder de controlo, que consiste na perceção de domínio que o indivíduo exerce sobre as crenças de controlo (Ajzen, 2002). Assim, os resultados sugerem que se o fumador acreditar que a sua dependência nicotínica é forte, menor será a perceção de controlo para deixar de fumar. De facto, a literatura sugere uma correlação positiva entre processos autorregulatórios (e.g. autoeficácia para deixar de fumar) e o controlo percebido (Schnoll et al., 2011). Neste sentido, será importante que a intervenção comportamental inclua o desenvolvimento de competências de autoeficácia na cessação tabágica.

Este estudo apresenta algumas limitações, nomeadamente, pelo facto dos fumadores serem jovens (idade inferior a 40 anos) e de $60 \%$ ter já efetuado uma tentativa para deixar de fumar tratando-se assim duma amostra muito motivada para deixar de fumar. Estudos longitudinais avaliando a estabilidade do constructo e dos resultados do instrumento, bem como a reação com outras medidas de dependência nicotínica, stresse, e coping, são importantes.

Assim, a versão adaptada do QCP-DF reúne as condições necessárias para a sua aplicação em fumadores, bastando o uso das medidas diretas, e poderá ser uma escala útil em termos de avaliação psicológica e de avaliação de resultados na intervenção na cessação tabágica (Casullo, 2009). Este instrumento (Anexo 1) poderá ainda ser uma ferramenta importante na avaliação dos preditores envolvidos na mudança do comportamento tabágico e servir para futuras avaliações das intervenções preventivas não só ao nível dos resultados mas também ao nível do processo (Font-Mayolas, Gras, \& Planes, 2013). 


\section{Referências}

Ajzen, I. (1985). From intentions to actions: A theory of planned behavior. In J. Kuhi \& J. Beckmann (eds.), Action control: From cognition to behavior (pp. 11-39). Heidelberg: Springer.

Ajzen, I. (1991). The theory of planned behaviour. Organizational Behavior and Human Decision Processes, 50, 179-211.

Ajzen, I. (2002). Constructing a TPB Questionnaire: Conceptual and Methodological Considerations. Retirado de http://www.apcc.online.pt/upload/membro.id/f icheiros/i005562.pdf.

Armitage, C. J. (2004). Evidence that implementation intentions reduce dietary fat intake: A randomized trial. Health Psychology, 23, 319-323.

Armitage, C. J., \& Arden, M.A. (2008). How useful are the stages of change for targeting interventions? Randomized test of a brief intervention to reduce smoking. Health Psychology, 27, 789-798.

Armitage, C. J., \& Conner, M. (2001). Efficacy of the theory of planned behaviour: A metaanalytic review. British Journal of Social Psychology, 40, 471-499.

Bardin, L. (2008). Análise de conteúdo. Lisboa: Edições 70.

Broadbent, E., Petrie, K., Main, J., \& Weinman, J. (2006). The brief illness perception questionnaire. Journal of Psychosomatic Research, 60, 631-637.

Casullo, M. M. (2009). La evaluación psicológica: modelos, técnicas y contextos. Revista Iberoamericana de Diagnóstico y Evaluación - e Avaliação Psicológica, 27(1), 9-28.

Cini, F. P., Flores, A. G., \& Pannuti, C. M. (2012). Dependência nicotínica em pacientes da Clínica Odontológica. Pesquisa Brasileira em Odontopediatria e Clínica Integrada, João Pessoa, 12(1), 99-105.

Costa, V., \& Pereira, M. G. (2015). Developing and psychometric testing of a Diabetes scale on Beliefs about Self-Monitoring of Blood Glucose. Manuscript submitted for publication.
Côté, F., Godin, G., \& Gagné, C. (2004). Identification of factors promoting abstinence from smoking in a cohort of elementary school children. Preventive Medicine, 39, 695-703.

Courneya, K. S., \& McCauley, E. (1995) Cognitive mediators of the social influence exercise adherence relationship: A test of the theory of planned behavior. Journal of Behavioral Medicine, 18, 499-515.

Dalum, P., Schaalma, H., \& Kok, G. (2012). The development of an adolescent smoking cessation intervention - an intervention mapping approach to planning. Health Education Research, 27(1), 172-181.

de Vries, H., Eggers, S. M., \& Bolman, C. (2013). The role of action planning and plan enactment for smoking cessation. BMC Public Health, 13(1), 393.

Dohnke, B., Weiss-Gerlach, E., \& Spies, C. (2011). Social influences on the motivation to quit smoking: Main and moderating effects of social norms. Addictive Behaviors, 36(4), 286-293.

Duarte, M. D. L. C., Campos, L. M. P., Monteiro, L., \& Oliveira, M. C. (2015). Grupo de tabagismo em um ambulatório de saúde mental no rio grande do sul. Revista Contexto \& Saúde, 14(27), 75-80.

Echer, I., Almeida, A., Lukasewicz, A., \& Lucena, A. (2001). Tabagismo em uma escola de enfermagem do sul do Brasil. Texto \& Contexto Enfermagem, 20(1), 152-159.

Ferreira, G., \& Pereira, M. G. (2014). Validation of the Questionnaire of Planned Behavior on Diabetes - Physical activity. Psicologia, Saúde \& Doenças, 15, 409-426.

Ferreira, G., \& Pereira, M. G. (2016). Physical activity: The importance of the extended theory of planned behavior, in type 2 diabetes patients. Journal of Health Psychology. doi:10.1177/1359105315626787

Ferreira, P. L., Quintal, C., Lopes, \& Taveira, N. (2009). Teste de dependência à nicotina: Validação linguística e psicométrica do teste de Fagerström. Dependência Tabágica, 27(2), 37-56.

Figueiras, M. J., \& Alves, N. C. (2007). Lay perceptions of serious illnesses: An adapted Vol.1 1 107-122 2017 
version of the Revised Illness Perception Questionnaire (IPQ-R) for healthy people. Psychology and Health, 22, 143-158.

Fila, S., \& Smith, C. (2006). Applying the Theory of Planned Behavior to healthy eating behaviors in urban Native American youth. International Journal of Behavioral Nutrition and Physical Activity, 3(1), 11-21.

Font-Mayolas, S., Gras, M. E., \& Planes, M. (2013). El proceso de valoración de programas preventivos en drogodependencias. Revista Iberoamericana de Diagnóstico y Evaluación - e Avaliação Psicológica, 35, 183-199.

Francis, J. J., Eccles, M. P., Johnston, M., Walker, A., Grimshaw, J., Foy, R.,... \& Bonetti, D. (2004). Constructing questionnaires based on the theory of planned behaviour: A manual for health services researchers. Retirado em novembro 20, 2007 do site: http://people.umass.edu/aizen/tpb.ht.

French, D. P., Sutton, S. R., Hennings, S. J., Mitchell, J., Wareham, N. J., Griffin, S.,... Kinmonth, A. L. (2005). The importance of affective beliefs and attitudes in the theory of planned behaviour: Predicting intentions to increase physical activity. Journal of Applied Social Psychology, 35, 1824-48.

Gollwitzer, P. M. (1999). Implementation intentions. Strong effects of Simple Plans. American Psychologist, 54, 493-503.

Heatherton, T. F., Kozlowsk, L. T., Frecker, R. C., \& Fagerstrom, K. O. (1991). Test for Nicotine dependence: a revision of the Fagerstrom tolerance questionnaire. British Journal of Addictions, 86, 119 -1127.

Hilberink, S. R., Jacobs, J. E., Schlösser, M., Grol, R. P., \& de Vries, H. (2006). Characteristics of patients with COPD in three motivational stages related to smoking cessation. Patient Education and Counseling, 61, 449-457.

Hill, A. J., Boudreau F., Amyot E., Déry D., \& Godin, G. (1997). Predicting the stages of smoking acquisition according to the theory of planned behavior. Journal of Adolescent Health, 21,107-115.

Homish, G., \& Kenneth L. (2005). Spousal influence on smoking behaviors in a US community sample of newly married couples. Social Science \& Medicine, 61, 2557-2567. Revista Iberoamericana de Diagnóstico y Evaluación - e Avaliação Psicológica. RIDEP · N43 · Vol.1 · 107-122 2017
Iacobucci, D. (2001). Factor Analysis. Journal of Consumer Psychology's, 10(12), 75-82.

Kovač, V. B., Rise, J., \& Moan, I. S. (2010). From intentions to quit to the atual quitting process: The case of smoking behavior in light of the TPB. Journal of Applied Biobehavioral Research, 14(4), 181-197.

Kraft, P., Rise, J., Sutton, S., \& Roysamb, E. (2005). Perceived difficulty in the theory of planned behaviour: Perceived behavioural control or affective attitude. British Journal of Social Psychology, 44, 479-496.

Lancaster, T. (2011). The role of doctors in smoking cessation: Evidence from the Cochrane Library. South African Heart Journal, 8(1), 24-27.

Mermelstein, R., Cohen, S., Lichtenstein, E., Baer, J., \& Kamarck, T. (1986). Social support and smoking cessation and maintenance. Journal of Consulting and Clinical Psychology, 54, 447-453.

Michie, S., Churchill, S., \& West, R. (2011). Identifying evidence-based competences required to deliver behavioural support for smoking cessation. Annals of Behavior Medicine, 4l(1), 59-70.

Moan, I. S., \& Rise, J. (2005). Quitting smoking: Applying an extended version of the theory of planned behavior in predicting intention and behavior. Journal of Applied Biobehavioral Research, 10, 39-68.

Norman, P., Conner, M., \& Bell, R. (2000). The theory of planned behaviour and exercise: Evidence for the moderating role of past behavior. British Journal of Health Psychology, 5, 249-61.

Nunnally, J. C. (1978). Psychometric theory (2nd ed.). New York: McGraw Hill.

Park, E., Tudiver, F., Schultz, J., \& Campbell, T. (2004). Does enhancing partner support and interaction improve smoking cessation? A meta-Analysis. Annals of Family Medicine, 2(2), 170-174.

Piasecki, T. M., Fiore, M. C., McCarthy, D. E., \& Baker, T. B. (2002). Have we lost our way? The need for dynamic formulations of smoking relapse proneness. Addiction, 97, 1093-1108.

Rise, J., Kovac, V., Kraft, P., \& Moan, I. (2008). Predicting the intention to quit smoking and 
quitting behaviour: Extending the theory of planned behaviour. British Journal of Health Psychology, 13(2), 291-310.

Rodrigues, A. (2009). Preditores da intenção e do uso de Protetor Solar. Tese de Mestrado. Universidade do Minho. Braga, Portugal

Rossaneis, M. A., \& Machado, R. C. B. R. (2012). Cessação do tabagismo em pacientes assistidos em um ambulatório de tratamento de dependência do tabaco. Ciência, Cuidado e Saúde, 10(2), 306-313.

Sattler, A. C., \& Cade, N. V. (2013). Prevalência da abstinência ao tabaco de pacientes tratados em unidades de saúde e fatores relacionados. Ciência \& Saúde Coletiva, 18(1), 253-264.

Schane, R. E., Ling, P. M., \& Glantz, S. A. (2010). Health effects of light and intermittent smoking a review. Circulation, 121(13), 1518-1522.

Schnoll, R. A., Martinez, E., Tatum, K. L., Glass, M., Bernath, A., Ferris, D., \& Reynolds, P. (2011). Increased self-efficacy to quit and perceived control over withdrawal symptoms predict smoking cessation following nicotine dependence treatment. Addictive Behaviors, 36(1), 144-147.

Sheeran, P., Milne, S., Webb, T. L., \& Gollwitzer, P. M. (2005). Implementation intentions and health behaviour. In M. Conner \& P. Norman (eds.), Predicting health behaviour (pp. 276323). Berkshire, England: Open University Press.

Sniehotta F. F., Scholz U., \& Schwarzer R. (2006). Action plans and coping plans for physical exercise: A longitudinal intervention study in cardiac-rehabilitation. British Journal of Health Psychology, 11, 23-37.

Sniehotta, F. F. (2009). Towards a theory of intentional behaviour change: Plans, planning, and self-regulation. British Journal of Health Psychology, 14, 261-273.

Souza, E. S. T. D., Crippa, J. A. S., Pasian, S. R., \& Martinez, J. A. B. (2009). Factorial structure of the Brazilian version of the Modified Reasons for Smoking Scale. Revista da Associação Médica Brasileira, 55(5), $557-$ 562.
Sutton, S. (2010). Using social cognition models to develop health behaviour interventions. Health Psychology, 122-132.

Topa, G., \& Moriano, J. A. (2010). Theory of planned behavior and smoking: Meta-analysis and SEM model. Substance Abuse and Rehabilitation, 1, 23-33.

Veloso, N. S., Rodrigues, C. A. Q., de Souza Leite, M. T., Ottoni, J. L. M., do Carmo Veloso, G. C., Rodrigues, R. M., \& Bandeira, G. A. (2011). Tabagismo: A percepção dos fumadores em um grupo de educação em saúde. Revista Brasileira de Medicina de Família e Comunidade, 6(20), 193-198.

Zhou, X., Nonnemaker, J., Sherrill, B., Gilsenan, A.W., Coste, F., \& West, R. (2009). Attempts to quit smoking and relapse: Factors associated with success or failure from the attempt cohort study. Addiction Behavior, 34(4), 365-373. 


\section{Anexo 1}

Versão Final do Questionário do Comportamento Planeado-Deixar de Fumar (QCP-DF): Medidas Diretas

-Intenção:

1. Eu tenciono deixar de fumar nos próximos 6 meses

Discordo Totalmente $\quad \begin{array}{llllll}1 & 2 & 3 & 4 & 5 & \text { Concordo Totalmente }\end{array}$

- Atitude face ao Comportamento

2. Não fumar todos os dias da semana nos próximos 6 meses é:

$\begin{array}{lllllll}\text { a) Mau } & 1 & 2 & 3 & 4 & 5 & \text { Bom } \\ \text { b) Muito inútil } & 1 & 2 & 3 & 4 & 5 & \text { Muito útil } \\ \text { c) Muito incómodo } & 1 & 2 & 3 & 4 & 5 & \text { Muito cómodo } \\ \text { d) Muito perigoso } & 1 & 2 & 3 & 4 & 5 & \text { Muito saudável }\end{array}$

- Normas Subjetivas

3. a) A maioria das pessoas que são importantes para mim pensam que eu

$\begin{array}{llllll}\text { Devia } & 1 & 2 & 3 & 4 & 5\end{array}$ Não devia deixar de fumar nos próximos 6 meses

b) $\mathrm{O}$ meu médico pensa que eu

$\begin{array}{llllll}\text { Devia } & 1 & 2 & 3 & 4 & 5\end{array}$ Não devia deixar de fumar nos próximos 6 meses

c) $\mathrm{O}$ (a) meu(minha) companheiro(a) pensa que eu

$\begin{array}{llllll}\text { Devia } & 1 & 2 & 3 & 4 & 5\end{array}$ Não devia deixar de fumar nos próximos 6 meses

d) A minha família pensa que eu

$\begin{array}{llllll}\text { Devia } & 1 & 2 & 3 & 4 & 5\end{array}$ Não devia deixar de fumar nos próximos 6 meses

- Crenças de Controlo Percebido

Finalmente para aceder às crenças de controlo percebido (perceived behavioural control) foram incluídos alguns itens que avaliavam a capacidade para deixar de fumar do indivíduo.

4. a) Deixar de fumar nos próximos 6 meses é uma tarefa...

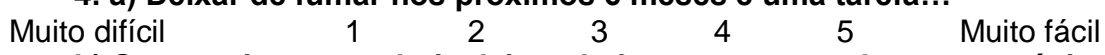

b) Se eu quisesse poderia deixar de fumar, por completo, nos próximos 6 meses.

$\begin{array}{lllllll}\text { Discordo totalmente } & 1 & 2 & 3 & 4 & 5 & \text { Concordo totalmente }\end{array}$

c) Quanto controlo acredita ter sobre o modo eficaz de deixar de fumar nos próximos 6 meses?

$\begin{array}{lllllll}\text { Nenhum controlo: } & 1 & 2 & 3 & 4 & 5 & \text { Completo controlo }\end{array}$

d) Deixar de fumar nos próximos 6 meses, por completo, depende apenas de mim.

$\begin{array}{lllllll}\text { Discordo totalmente } & 1 & 2 & 3 & 4 & 5 & \text { Concordo totalmente }\end{array}$

- Planeamento do Coping

5. Para os próximos 6 meses tenho um plano em relação a...

a) Deixar de fumar

Nada Verdadeiro $1 \quad 2 \quad 3 \quad 4 \quad 5$ Completamente Verdadeiro

b) Conviver com amigos fumadores

Nada Verdadeiro $1 \quad 2 \quad 3 \quad 4 \quad 5$ Completamente Verdadeiro

c) Antecipar situações que provoquem ansiedade

Nada Verdadeiro $1 \quad 2 \quad 3 \quad 4 \quad 4 \quad 5$ Completamente Verdadeiro

d) Ao que vou fazer para não tomar café

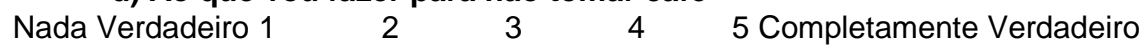

\section{- Planeamento da Ação}

6. Para os próximos 6 meses tenho um plano detalhado quanto...

a) Ao que fazer para organizar o meu tempo para não fumar

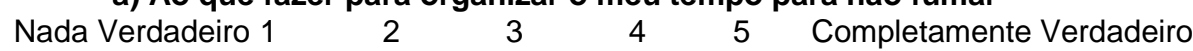

b) Ao que fazer para não fumar

$\begin{array}{llllll}\text { Nada Verdadeiro } 1 & 2 & 3 & 4 & 5 & \text { Completamente Verdadeiro }\end{array}$

$\begin{array}{lllll}\text { c) A não comprar cigarros } & & & \\ 3 & 4 & 5 & \text { Completamente Verdadeiro }\end{array}$

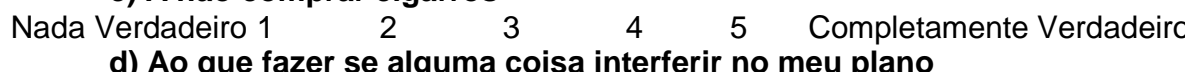

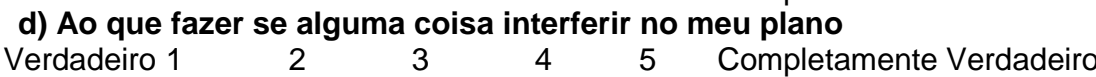

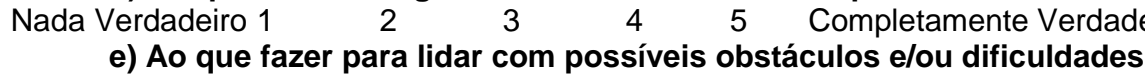

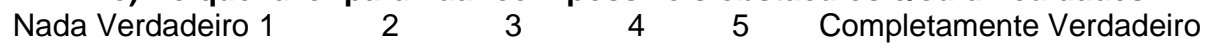

